\title{
The new fluorescent probes on the block
}

The combination of environmentally sensitive small organic dyes with single-chain antibodies creates an extendable family of new fluorescent probes.

Fluorescence imaging has become a methodological pillar of biological investigation. One reason for this is the explosion in the number and variety of fluorescent probes available for researchers. In spite of this, researchers continue to push the boundaries of what is possible with the available probes.

This continuing need for new probes with new characteristics has prompted the US National Institutes of Health to fund centers devoted to probe development. One of these centers is the Molecular Biosensor and Imaging Center at Carnegie Mellon University where Alan Waggoner, who has been developing fluorescent probes for years, is the director.

Although much fluorescent probe development and use has been focused on fluorescent proteins, Waggoner has concentrated on developing small chemical fluo-

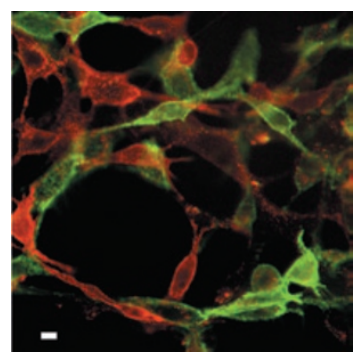

Figure 1 | Visualization of cell-surface receptors tagged with two different FAPs and exposed to green and red fluorogens. Scale bar, $10 \mu \mathrm{m}$. Reprinted from Nature Biotechnology.

rophores such as the popular Cy3 and Cy5 dyes. Small chemical dyes are ideal for some applications, but the difficulty in using them to selectively label proteins in living cells is a drawback for many applications. Rather, fluorescent proteins have proven very popular for these applications.

Most fluorescent proteins, however, have the drawback that labeling requires creation and expression of hybrid fusion proteins that are constitutively fluorescent. Waggoner and his coworkers wanted a way to label target proteins in living cells at a time and place chosen by the investigator. Their concept was to combine environmentally sensitive dyes called fluorogens that are nonfluorescent in solution with protein fusion tags that can bind the dyes and induce a strong fluorescent signal. Thus, an investigator could visualize a target protein containing a tag at a desired time by applying the fluorogen to the cell.

They picked human single-chain antibodies (scFvs) as their protein tag platform to create fluorogen activating proteins (FAPs) and then selected candidate fluorogens. "We went into our treasure chest of dyes that we have had for many years and picked some that we thought we could combine with the single-chain antibodies and get substantial fluorescent signals," says Waggoner.

Because of their extensive experience with small molecule dyes, the first one they picked, TO1, worked. This fluorogen was known to become highly fluorescent upon binding

\section{GLYCOBIOLOGY}

\section{SUGARS DIRECT STEM CELL HOMING}

\section{Addition of fucose to a cell-surface glycoprotein receptor demonstrates the power of glycan engineering to direct homing of adult stem cells to a tissue of choice.}

Stem cells hold great promise for the repair of damaged organs, but they first need to be delivered to where they are needed, ideally via the bloodstream. In an effort to meet this delivery challenge for skeletal diseases such as osteoporosis, Robert Sackstein and his team at the Biomedical Research Institute of Brigham and Women's Hospital sought a method to direct boneforming stem cells to bone.

The researchers have a long history of working with hematopoeitic stem cells (HSC), which efficiently migrate to the marrow, the portal of entry to bone tissue. They previously identified the cell-surface receptor on these HSCs, called HCELL, a glycosylated variant of the transmembrane protein CD44, responsible for the homing of the cells from the blood stream to bone marrow. CD44 is expressed on every cell type but varies in its glycosylation pattern.

HCELL on HSCs carries a tetrasaccharide containing a sialyl acid and fucose that is specifically recognized by E-selectin, a carbohydrate binding protein on the surface of bone marrow blood vessels. This ligand-receptor interaction is strong and specific, promoting effective recruitment of the HSCs. Unfortunately HSCs do not contribute to bone formation and are thus not suitable for any therapeutic application that seeks to increase bone mass.

Mesenchymal stem cells (MSC) are the prototypical osteoprogenitor cells and are therefore the optimal candidates for growing bone, but these stem cells do not naturally home to bone.

To discover what prevented MSCs from finding their way to bone tissue, Sackstein needed to find out what exactly was missing on the CD44 of MSCs. A panel of antibodies that had been generated to analyze specific tetrasaccharide modifications on CD44 of HSCs provided the answer. MSC did have sialyated CD44, but it was lacking the fucose.

The researchers wanted to supply these cells only with the specific glycan that was missing, and to this end they developed a protocol that would specifically fucosylate CD44 on MSCs in vitro. It proved more challenging than anticipated because the reported conditions under which the enzyme was active in vitro, especially the presence of divalent cations, are toxic to the cells. Sackstein summarizes several years of effort: "We specifically formulated the enzyme and the reaction conditions to allow the ex vivo glycan engineering on the cell surface without harming the cells". 
DNA. Using selection and directed evolution of the scFvs and chemical modification of TO1 they obtained a high affinity scFv-dye combination that produced a 2,600-fold fluorescence enhancement of the green-emitting fluorogen.

One of their goals is to create a library of scFv-fluorogen pairs with different spectral and physical properties. As a first step on this road they used a red fluorogen, MG, and selected FAPs that induced fluorescence up to 18,000-fold. Additional colors are likely on the way.

Both TO1 and MG are cell-impermeable, which is ideal for selectively labeling functional cell-surface receptors (Fig. 1). To complement the cell-surface labeling they also developed a cell-permeable ester of MG that labels proteins inside the cell. According to Waggoner, "While chemical modification of TO1 reduced DNA binding and nonspecific binding to cell material, MG is more suited for intracellular labeling due to its lower DNA binding." The low fluorescence due to nonspecific binding to cellular proteins may be an advantage over a different fluorogen system based on a tetracysteine protein tag and biarsenical dyes. In contrast to the tetracysteine tag, intracellular labeling with FAPs is now limited to the cell lumen because scFvs are only partially functional in the reducing environment of the cytoplasm, but Waggoner's group thinks this limitation will likely be overcome in the future.

The flexible design of their FAP system suggests that the capabilities of this class of probes have only begun to be explored, and it will likely become a valuable tool for dissecting cellular pathways.

\section{Daniel Evanko}

\section{RESEARCH PAPERS}

Szent-Gyorgyi, C. et al. Fluorogen-activating single-chain antibodies for imaging cell surface proteins. Nat. Biotechnol. 26, 235-240 (2008).

Once they added fucose to CD44, the modified MSC did indeed home to bone very efficiently and began to differentiate into bone cells. Expression of the modified glycan was temporary-presumably lost through normal cell-surface turnover of CD44.

The targeting of MSC to bone is just one application; the real power of glycan engineering is that it can steer any stem cell to any tissue type. As Sackstein explains, "We are inducing a temporal homing capability in the cell that will get it to where it needs to go and then the cell reverts to its native surface molecule configuration to proceed down its normal path of development."

CD44 is expressed on all stem cells, and the challenge of course lies in identifying exactly which glycan modification is missing to target the tissue of interest, then optimizing the enzymatic conditions needed to add this carbohydrate. The Sackstein lab is currently working on developing glycosyltransferases that will modify any cell surface under conditions that do not evoke cell toxicity.

These tools will prove valuable in controlling the homing of stem cells.

Nicole Rusk

RESEARCH PAPERS

Sackstein, R. et al. Ex vivo glycan engineering on CD44 programs human multipotent mesenchymal stromal cell trafficking to bone. Nat. Med. 14, 181-187 (2008).

\section{NEWS IN BRIEF}

\section{RNA INTERFERENCE}

\section{siRNAs unveil HIV's helpers}

With its mere 15 self-encoded proteins, HIV relies heavily on the host cell for infection - but to date only some of these host factors have been identified. Brass et al. expand this list to over 250 proteins by infecting cells that were transfected with a genome-wide small interfering RNA library. They identified proteins needed for viral entry and then used the supernatants from this screen with fresh reporter cells to assay for late-acting factors. Brass, A.J. et al. Science, published online 10 January 2008.

\section{CHEMICAL BIOLOGY}

\section{Profiling kinase inhibitors}

Kinases are important drug targets, but kinase inhibitors often exhibit promiscuous activities and cause off-target effects. Karaman et al. present a comprehensive evaluation of the selectivity of 38 kinase inhibitors across a panel of 317 kinases, representing about $50 \%$ of the human kinome. This resource should help researchers understand structure-activity relationships and develop more selective kinase inhibitors. Karaman, M.W. et al. Nat. Biotechnol. 26, 127-132 (2008).

\section{PROTEOMICS}

\section{Quantitative 2D gel electrophoresis}

Robust methods for quantitative analysis of two-dimensional gel electrophoresis data are lacking. Morris et al. now describe an algorithm called Pinnacle for quantifying protein spots, based on aligning multiple gels, computing an 'average gel' and detecting spot 'pinnacles' above a given threshold. Pinnacle outperforms two commercially available software packages and should be well-suited for large-scale proteomics studies. Morris, J.S. et al. Bioinformatics, published online 14 January 2008.

\section{VIROLOGY}

\section{Prion detection in organotypic brain slices}

The ultimate confirmation of prion disease is the bioassay that involves inoculating animals with a sample from a diseased animal and waiting to see if the inoculated animals develop the disease. This is slow and expensive. Falsig et al. now present a prion organotypic slice culture assay (POSCA) that uses mouse cerebellar slices to amplify prion protein in 35 days. They used POSCA to detect prions in both cow and sheep samples. Falsig, J. et al. Nat. Neurosci. 11, 109-117 (2008).

\section{CELL BIOLOGY}

\section{Nano-sized electrochemical probes}

Electrochemical measurements of cells provide information about their redox properties, but obtaining these measurements is very difficult and disruptive to the cell. Sun et al. now describe nanometer-sized electrochemical probes that can penetrate mammalian cell membranes without causing any damage. They were able to quantitatively measure intracellular redox properties and charge transfer kinetics.

Sun, P. et al. Proc. Natl. Acad. Sci. USA 105, 443-448 (2008). 